\title{
An intelligent technical analysis using neural network
}

\author{
Reza Raei $^{\mathrm{a}}$, Shapour Mohammadi ${ }^{\mathrm{a}}$ and Mohammad Mehdi Tajik ${ }^{\mathrm{a}^{*}}$
}

${ }^{a}$ Department of Management Science, University of Tehran, Tehran, Iran

\begin{tabular}{l}
\hline A R T I C L E I N F O \\
\hline Article history: \\
Received October 162010 \\
Received in revised form \\
14 February 2011 \\
Accepted 14 February 2011 \\
Available online \\
15 February 2011 \\
\hline Keywords: \\
Technical Analysis \\
Generalized regression neural \\
network \\
Volume adjusted moving average \\
Ease of movement \\
Stock market
\end{tabular}

\section{A B S T R A C T}

Technical analysis has been one of the most popular methods for stock market predictions for the past few decades. There have been enormous technical analysis methods to study the behavior of stock market for different kinds of trading markets such as currency, commodity or stock. In this paper, we propose two different methods based on volume adjusted moving average and ease of movement for stock trading. These methods are used with and without generalized regression neural network methods and the results are compared with each other. The preliminary results on historical stock price of 20 firms indicate that there is no meaningful difference between various proposed models of this paper.

Stock market

\section{Introduction}

During the past few decades, there have been significant attempts to develop practical methods for predicting stock market behaviors based on either fundamental or technical methods. In fundamental method, we are interested in studying the impact of micro or macroeconomics conditions along with company's assets values for stock price predictions. Many fundamental methods rely on past performance of the stock market but there is an argument that past performance does not necessarily guarantee future success. On the other hand, in an efficient market, stock price normally includes all fundamental facts and we could hardly benefit from the news for bargain hunting solely based on the existing news. The other method for stock market price prediction is called technical analysis where we rely on different technical indicators such as stock price, volume of trading per day, etc (Malkiel, 1995). There are different studies on technical analysis (TA) where some suggest that TA could be effectively used for stock price prediction (Blume \& O`Hara, 1994) and the others do not. There are different scientific methods used to derive technical rules for stock market predictions such as metaheuristic approaches. Allen and Karjalainen (1999) used genetic algorithm to find technical trading

* Corresponding author. Tel: +98912-2079320

E-mail addresses: mmtajik.broker@gmail.com (M. M. Tajik) 
rules. Acheist (1995) in his book reported that many analysts and financial firms used TA along with fundamental methods for predicting stock market. There are also many people who believe that TA must be used along with fundamental analysis for investment decisions. In fact, there may be several buying opportunity on stocks which may fundamentally sound but we need some technical signals to find the right time for buying opportunities (Arms, 1996).

Trading volume is one of the most important key TA indicators and there has been different studies to report that an unusual increase on volume with a positive stock momentum could suggest a strong buying opportunity (Lee \& Swaminathan, 2000). There is a more sophisticated TA indicator based on the trading volume called EuiVolume (EV) which shows the trading volume in time horizon (Arms, 1996). Neural network is another useful tool to study the trend on stock price. There are literally different neural network methods for stock price prediction (see Chavarnakul, T., \& Enke, 2008 for a comprehensive discussion).

The study of this paper aims to use generalized regression neural network (GRNN) with EV for stock price prediction. We also study volume adjusted moving average (VAMA) and ease of movement (EMA) which are normally used along with EV method for stock price prediction. In fact, VAMA replaces time with trading volume in moving average for stock trading. Also, EMV shows the price changes against the trading volume which is an interesting indicator for buying/selling strategies. Our primary purpose is to see whether the GRNN could help evaluating EMV or VAMA signals. For VAMA, we use GRNN method to predict stock price and EMV we implement GRNN to examine whether we could use EMV for decision making.

This paper is organized as follows. We give a brief explanation of different methods in section 2 . Section 3 is dedicated to the details of the implementation of our hybrid methods and the results of the proposed model is used for some real-world data. Finally, conclusion remarks are given in section 4 to summarize the contribution of this paper.

\section{Notations and definitions}

In this section we briefly explain the necessary notations and deifinition used for the proposed methods of this paper.

\subsection{EquiVolume}

This indicator explains the relationship between the price volatility and trading volume (Arms, 1996). Fig. 1 shows the characteristics of Equivolume based on maximum, minimum and trading volume.

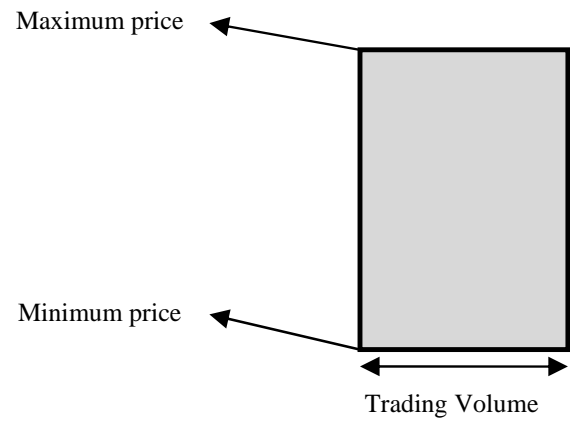

Fig. 1. Characteristics of EquiVolume

As we can observe from Fig 1, this indicator does not depend on time and rely only on price and trading volume. In other word, EquiVolume is based on trading volume and we believe that the trading volume reflects the price jump better than time. 


\subsection{Volume adjusted moving average}

As already explained, volume adjusted moving average (VAMA) is considered better than the simple moving average since it gives more weight to trading volume in its computations. In VAMA, the trades with higher volumes are significantly more meaningful indicators than trades with lower volumes. VAMA gives buying signal once price curve is located higher than VAMA curve and it gives selling price when price is located under VAMA.

\subsection{Ease of movement}

This indicator shows the relationship between price movement and trading volume and indicates the volume of trades needed for price movement. This indicator is high when the price goes up with low volume. This indicator gives buying signal when the price goes up and the indicator is above zero. To calculate this ratio we first calculate box ratio (BR) and midpoint move (MPM) as follows,

$$
\begin{aligned}
& B R_{t}=\frac{V_{t}}{H P_{t}-L P_{t}}, \\
& M P M_{t}=\left(\frac{H P_{t}+L P_{t}}{2}\right)-\left(\frac{H P_{t-1}+L P_{t-1}}{2}\right) .
\end{aligned}
$$

where $B R_{t}$ is the box ratio, $V_{t}$ is the trading volume, $H P_{t}$ and $L P_{t}$ are high and low price at time $t$. Also $M P M_{t}$ is the midpoint move at time $t, H P_{t-1}$ and $L P_{t-1}$ are high and low price at time $t-1$. Therefore, ease of movement at time $t$ is calculated as follows,

$$
\text { Ease of Movement } t_{\mathrm{t}}=\frac{M P M_{t}}{B R_{t}} \text {. }
$$

\subsection{Neural network}

Neural network has been one of the most important techniques for forecasting stock prices. The basic idea of neural network is to build a system based on how a human brain works. In other words, there is a relationship between two layers of input and output with some neurons located in hidden layers. Generalized regression neural network (GRNN) is a special form of neural network, which is used for stock prediction in financial markets. Specht (1991) first introduced the idea of GRNN, which is a one-way method with parallel structure. GRNN establishes a memory based on neural network, which stores dependent and independent data. GRNN uses an interval function to calculate the average weight of learning data (Heimes \& Van Heuveln, 1998). GRNN estimator is based on a nonlinear regression function, which uses the probability density function for the data. Let $x$ be an independent variable and $y$ be dependent variable. Then the conditional expected value of $y$ given $x$ is as follows,

$$
E(y \mid x)=\frac{\int_{-\infty}^{+\infty} y f(x, y) d y}{\int_{-\infty}^{+\infty} f(x, y) d y} .
$$

Note that we can use Eq. (4) only when we know the distribution of $f(x, y)$. However, in most cases we normally do not know much about the distribution of this function and use historical data to 
find a suitable estimate using nonlinear estimators (Parzen, 1962). Let $x_{i}$ and $y_{i}$ be the sample size for $x$ and $y$, respectively. Therefore, we have,

$\hat{y}(x)=\frac{\sum_{i=1}^{n} y_{i} W\left(x, x_{i}\right)}{\sum_{i=1}^{n} W\left(x, x_{i}\right)}$,

where $W\left(x, x_{i}\right)=e^{-\left(D_{i}^{2} / 2 \sigma^{2}\right)}$ is the hidden layer of the first output, $D_{i}^{2}=\left(x_{i}-x\right)^{\prime}\left(x_{i}-x\right)$ and $\sigma$ is a given parameter which is considered to be equal to one in this study. Fig. 2 demonstrates the structure of the proposed GRNN of this paper.

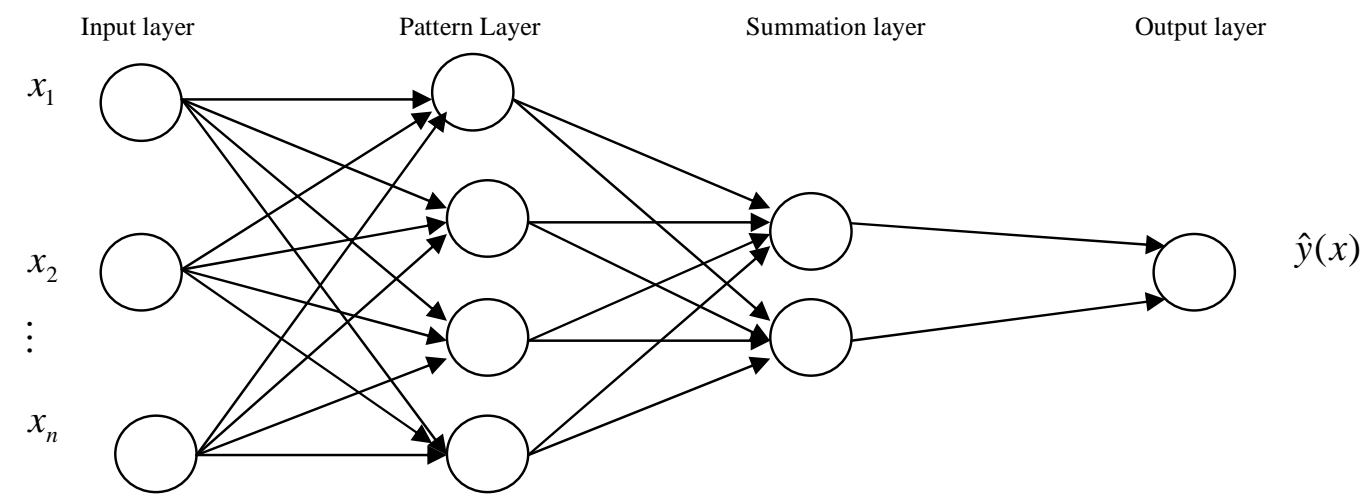

Fig. 2. GRNN architecture

As we can observe from Fig 2, there are four layers associated with the proposed GRNN and it is very similar to feed-forward networks but the operations are totally different. The first hidden layer computes $D_{i}^{2}$ for each $x_{i}$ and they are summed up through an exponential function. The pattern units generate $W\left(x, x_{i}\right)$ and they transfer them into hidden layers, which are used for final weighted estimations. The sum unit uses the first input for the sum of $y_{i} W\left(x, x_{i}\right)$ and the second input for $W\left(x, x_{i}\right)$. According to Specht (1991), GRNN provides better nonlinear estimations than other neural networks models. It can learn faster and normally yields lower residuals than other linear techniques. The proposed GRNN of this paper uses Gaussian distribution as follows,

$y(x)=\frac{\sum_{i=1}^{n} y_{i} e^{-D_{i}^{2} / 2 \sigma^{2}}}{\sum_{i=1}^{n} e^{-D_{i}^{2} / 2 \sigma^{2}}}$,

$D_{i}=\left(x-x_{i}\right)^{\prime}\left(x-x_{i}\right)$,

where $D_{i}$ computes the distance between the training and predicted points. Obviously, smaller $D_{i}$ yields bigger values for $D_{i}^{2} / 2 \sigma^{2}$ and larger values for $D_{i}$ yield smaller values for $D_{i}^{2} / 2 \sigma^{2}$. In this paper, two neural network systems are developed. The first neural network (NN) system uses two versions of GRNN model combined with future trading volume and price. The second GRNN model uses EMV for stock price prediction. Table 1 summarizes the input and the output parameters of the proposed model. 
Table 1

The characteristics of input and output parameters

\begin{tabular}{llll}
\hline $\begin{array}{l}\text { Neural } \\
\text { Network }\end{array}$ & Model & Input parameters & Output \\
parameters
\end{tabular}

We have trained our neural network models using the historical information of 20 actively trading firms on Tehran Stock Exchange (TSE) between March, 2005 and Feb, 2006 and the trained system were used from March, 2008 to Feb, 2009. Note that two major indexes of TSE namely Tehran stock price index (TEPIX) and Tehran stock dividend and price index (TEDPIX) did not have any special trend and somehow they had negative trends. In fact, these indexes had small positive trends during the first six months and they had negative trends during the second six months. The proposed models use $\sigma=1$ and the rate of trading volume (VR) is calculated as follows,

$V R_{t+1}=\frac{v_{t+1}}{\sum_{i=1}^{n} v_{i} / n}$,

where $v_{t+1}$ is the trading volume of the next business day and $v_{i}$ is the trading volume and $n$ is the number of business days. In addition, the return for the next business day is calculated as follows,

$R_{t+1}=\frac{C P_{t+1}-C P_{t}}{C P_{t}}$ 
where $R_{t}$ is the return at time $t, C P_{t}$ and $C P_{t+1}$ at time $t$ and $t+1$, respectively.

For the second neural network system, we only use one GRNN model stated as GRNN3 and the model has 17 input parameters.

The implementation of the proposed models of this paper first normalizes all the input data based on the well-known normal relationship as follows,

$$
z_{i}=\frac{x_{i}-\mu}{\sigma},
$$

where $z_{i}, x_{i}, \mu$ and $\sigma$ are the Gaussian variable, observation, mean and standard deviation of the data, respectively.

\section{Results}

In order to study the performance of the proposed models we have built two hypotheses. Based on the first hypothesis, we study whether the return of investment volume adjusted moving average (VAMA) with neural network is higher than the return of investment using VAMA without neural network.

The second hypothesis examines whether the return of investment using EMV with neural network is higher than the return of investment using direct implementation of EMV. One popular method to measure the relative strength of a method is to compare the mean square of errors as follows,

$$
\text { MSE }=\frac{\sum_{\mathrm{i}=1}^{\mathrm{n}}\left(\text { Actual }_{\mathrm{i}}-\text { Predition }_{\mathrm{i}}\right)^{2}}{\mathrm{n}},
$$

where $n$ is the number of periods. However, some people argue that MSE may not show the performance of the proposed models, properly (Pesaran \& Timmermann, 1995) and recommended to use significance number (SIGN) as an alternative to use (Leitch \& Tanner, 1991). In EMV, it is important to measure the performance of the predicted method against the actual data. We use the following to measure the percentage of positive or negative,

Percentage of Positive or negative $=\frac{\sum_{i=1}^{n} c_{i}}{n}$,

where $c_{i}=1$ is both predicted and actual data are either positive or negative and $c_{i}=0$, otherwise.

\subsection{Investment strategies}

The following four strategies are considered to measure the relative performance of the proposed models,

1. For VAMA with neural network, if the closing price of the period $t+1$ is more than the VAMA value for that period, we take a buying action. In addition, if the closing price of the period $t+1$ is less than the VAMA value for that period, we take a selling action. In other cases, we perform no buy/sell action. 
2. For simple VAMA we take similar action as we do in the previous strategy.

3. For simple MA, if the closing price of the period $t+1$ is more than the MA value for that period, we take a buying action. In addition, if the closing price of the period $t+1$ is less than the MA value for that period, we take a selling action. In other cases, we perform no buy/sell action.

4. In EMV, whenever the closing price is higher than EMV value, in above zero region, we take a buy action and if closing price is less than the EMV value, in below zero region, we take sell action. In other cases, we take no action.

Table 2 summarizes the results of the performance of all methods.

Table 2

The summary of the results

\begin{tabular}{lccc}
\hline Neural network model & MSE & SIGN & Percentage of positive or negative \\
\hline GRNN1 & 0.99272 & $65 \%$ & - \\
GRNN2 & 1.222165 & $61 \%$ & - \\
GRNN3 & 28.94863 & $57 \%$ & $71 \%$ \\
\hline
\end{tabular}

The performance of the proposed models of VAMA derived from neural network, VAMA without using neural network and simple MA are 11.5\%, 10.5\% and 9.3\%, respectively. We have performed an ANOVA analysis to verify whether there is any meaningful difference among the mean of these three methods. Table 3 summarizes the results of the ANOVA test.

Table 3

The ANOVA test results

\begin{tabular}{lccccc}
\hline & Sum of squares & df & Mean square & F & Sig. \\
\hline Between groups & 5132.745 & 2 & 2566.372 & 0.023 & 0.977 \\
Within groups & 6348138.021 & 57 & 111370.842 & & \\
\hline Total & 6353270.766 & 59 & & & \\
\hline
\end{tabular}

Based on the results of Table 3 we realize that Sig=0.977 is significantly higher than $5 \%$ and we can conclude, with the probability of $95 \%$, that the there is no meaningful difference among three methods.

We have compared the performance of EMV for our case study in two cases of with and without using neural network and the returns were $-14.7 \%$ and $-34.4 \%$, respectively. We also performed a $t$ student test as well as sig. test for the returns of two methods and they both indicated that, with a probability of 95\%, there was no difference between two methods.

\section{Conclusions}

In this paper, we have examined different technical methods for the best stock trading. The proposed models of this paper use a combination of different versions of moving average with neural network to build some hybrid methods for price predictions. We have used generalized regression neural network with different techniques, and the proposed models were trained using the information of 
one-year data and then they were used for price prediction for another one-year period. We established different tests to see whether there is any meaningful difference on the return of investment based on each of these methods. The results indicated that the implementation of the special form of neural network did not have any positive impact on the return of investment. The other observation was that the simple moving average performed better than EMV method.

\section{References}

Acheist, S. B. (1995). Technical analysis from A to Z: covers every trading tool from the absolute breadth index to the zig zag. Chicago: IL: Probus Publisher.

Al-Daoud, E. (2009). A comparison between three neural network models for classification problems. Journal of Artificial Intelligence , 2 (2), 56-64.

Allen, F., \& Karjalainen, R. (1999). Using genetic algorithms to find technical trading rules. Journal of Financial Economics , 51(2),245-271.

Arms, R. W. (1996). Trading without fear: eliminating emotional decisions with arms trading strategies. Toronto, Canada: John Wiley \& Sons, Inc.

Bauer, M. M. (1995). General Regression Neural Network for Technical Use. Master`s Thesis: University of Wisconsin-madison.

Blume, E. D., \& O`Hara, M. (1994). Market statistics and technical analysis: the role of volume. Journal of Finance , 49(1), 153-181.

Chavarnakul, T., \& Enke, D. (2008). Intelligent technical analysis based equivolume charting for stock trading using neural networks. Elsevier Ltd , 1004-1017.

Heimes, F., \& Van Heuveln, B. (1998). The normalized radial basis function neural network. In Proceedings of the 1998 IEEE international conference on systems, man, and cybemetics, (pp. 1609-1614). Sandiego, CA, USA.

Kaastra, I., \& Boyd, M. (1996). Designing a neural network for forecasting financial and economic time series. Neurocomputing , 215-236.

Lee, C. M., \& Swaminathan, B. (2000). Price momentum and trading volume. Journal of Finance , 55(5), 2017-2069.

Leitch, G., \& Tanner, J. E., (1991). Economic forecast evaluation: profits versus the conventional error measures. Model-Evaluation-and-Testing, 81(3), 580 - 90.

Malkiel, B. G. (1995). A random walk down wall street. New York: NY: Norton \& Co.

Parzen, E. (1962). On estimation of a probability density function and mode. Annal of Mathematical Statistics , 1065-1076.

Specht, D. F. (1991). A general regression neural network. IEEE Transactions on Neural Networks, 2(4), 568-576.

Pesaran, M. H. \& Timmermann, A., (1995). Predictability of Stock Returns: Robustness and Economic Significance, Journal of Finance, American Finance Association, 50(4), 1201-1228. 